Daniel Bell and Wang Pei, Just Hierarchy: Why Social Hierarchies Matter in China and the Rest of the World. Princeton: Princeton University Press, 202O. 288 pages, ISBN: 978-o691200897.

The core message of Daniel Bell and Wang Pei's 汪沛 new book, Just Hierarchy: Why Social Hierarchies Matter in China and the Rest of the World is clear, even elegantly so. They state: "Whatever the drawbacks of traditional forms of hierarchy, the effort to combat all forms of hierarchy is neither possible nor desirable" (p. 13). Furthermore, “...(t)he claim that morally justifiable social hierarchies should structure our social lives on an everyday basis, including our relations with loved ones. That's the claim we'd like to defend in this book" (p. 14). And defend they do, or at least attempt to. The result is, unfortunately, not as convincing as one might hope, particularly given that the conversation itself, in effect a softly spoken interrogation into the nature of human social interaction, is very relevant to the current moment in time, a moment when the power dynamics on a global stage are, indeed, shifting, and China's rise to prominence if not supremacy within the coming decades either forces us or provides an opportunity for us - depending on one's view - to imagine a different approach to social and political structure writ large. Adding to this now is the upheaval of COVID-19, coming itself amid a longer-term shift from global to local, internationalist to nationalist or even nativist politics, and we are faced as never before with the basic question of how to best interact with others, near and far. Bell and Wang's work responds directly to this question and at least furthers the conversation in some productive ways.

The problem is, in some respects, one of tone. On page 30 , for instance, amid a discussion of hierarchies as they are manifested in relationships between lovers, the authors make the following point: "We oppose any relationship that involves involuntary physical violence toward another person, no matter how loved the recipient" (p. 30)-similarly, in a discussion of parent-child relations and in mere parenthetical terms "including physical punishment, which we do not endorse" (p. 53). Gratifying as it might be to learn that the authors do not encourage domestic violence, the notion that a work as expansive as thisparticularly given that the expansiveness is covered in the relatively condensed space of only 206 pages - would also be a sort of "how to" for the modern world in terms of organizing human relationships - whether in the form of romantic partnerships, family, small social groups, larger organizations, governments, or national and global entities, in short, everyone everywhere-is a bit hard to accept. The first question that might come to mind is: Who are the authors to endorse (or not) anything in terms of social relationships? The answer is simply that the two are professors working in China (Bell at Shandong University and 
Tsinghua, Wang at Fudan University in Shanghai). Bell has published articles on related topics in the past, if by "related" we mean advocacy for a reappraisal or even critique of what has for decades passed as virtually universally accepted truth: that human relationships based on individual rights and extended to a larger system of liberal democracy fueled by capitalism is the path forward for all of humanity. Exposing that and related assumptions to careful scrutiny is certainly long overdue, and Bell has contributed significantly to this endeavor. In previous works, such as China's New Confucianism: Politics and Everyday Life in a Changing Society, though, Bell's territory was narrower, and more explicitly China focused, even if the implications of his work have always been a critique of Western-centric political thinking. ${ }^{1}$ This book, as the subtitle makes clear, is a full-on effort to include "the rest of the world," and therein lies the challenge that the work does not quite meet. The authors tackle their subjects bravely, even fearlessly, and their tone is authoritative ("we do not endorse") throughout. Their authority, however, is not entirely defensible given the highly disparate nature of the "fields" into which they almost meander. Bell and Wang are principally scholars of political philosophy, and it is in that area that their contribution is most notable. Put another way, it is one thing to opine on how hierarchies can, should, and should not work in parent-child relationships, and the implications of such relationships for larger political structures, essentially, Confucian social programming. To move into dynamics of human sexual experience (hierarchically speaking, "dominant" and "passive") seems to require some other mode of inquiry, not to mention academic literature review, and again the same as the topic shifts abruptly into regional and global politics, human-animal relations, artificial intelligence, and more. The scope of the discussion, in other words, is vast, and boldly so. It is undercut, though, by a sort of breezy style that suggests the relatively non-academic nature of the writing - the target audience is certainly general interest—and at the same time a questionable attempt to cover "everything" hierarchical, as though such an enterprise would be possible. Nonetheless, the authors proceed as though their pronouncements on this vast collection of topics is somehow warranted and worthy of consideration.

It is also important to note that Bell and Wang's work does not come from a vacuum, by any means. Principally, this discussion draws on contemporary philosopher Zhao Tingyang's 趙汀陽 promulgation of a revised Confucian “all under heaven" [tianxia 天下] in the contemporary world, one that extends far beyond its original Chinese origins, to be located in some super-governmental

1 Daniel Bell, China's New Confucianism: Politics and Everyday Life in a Changing Society (Princeton: Princeton University Press, 2008). 
entity with dominion over the entire world. The problem with Zhao's view, Bell and Wang observe, is that it equalizes all forms of mutual care across a geographic territory that is untenable and, in any event, not aligned with actual Confucian teaching. The authors go on (pp. 132-135) to amend Zhao's theory according to a Confucian approach predicated on "graded love," meaning a degree of commitment to those around us based on concentrically graduated circles extending from intimate, in which reciprocal action is most intense, to remote, where although responsibility remains to both the human and animal realms broadly speaking, it is undertaken with much less engagement and commitment. In this sense, Bell and Wang's book is a welcome step toward the practical application of the ideas of just hierarchical interactions, schematized, for instance, in terms of "strong" and "weak" reciprocity. This is arguably the core contribution of their work. They begin with the observation that technically, in terms of global political discourse, not to mention law (at least since the Peace of Westphalia of 1648), each state is an equal among others. This equality belies the essential fact, though, that some states are clearly stronger and more significant than others and, accordingly, have different responsibilities to the global order. The relationships, in short, are always hierarchical to some degree and should be recognized as such. The distinction in terms of reciprocity is as follows: essentially, in "weak" relationships, reciprocity exists, but it is fragile and narrowly understood, with each party to the relationship focused solely on what it can gain, meaning that after a shift in benefit, the relationship ceases to be desirable, thus even minor fluctuation can mean the demise of the relationship itself. "Strong" reciprocity, meanwhile, is more durable, defined as a relationship in which each side is aware of and attentive to the other's benefit, even when its own is occasionally minimized or entirely nonexistent. The idea is that a strong reciprocal relationship exists with a full view of a longer-term relationship, elastic enough in its connection to weather, so to speak, the vicissitudes of material and even psychological change over time. As the authors describe:

what counts as the interest of each state itself comes to be influenced, at least partly, by the interests (and culture and history) of the other state: There is mutual earning that affects how people think of their own interests and conceptions of the good life. (p. 107)

This is actually quite profound, as it pushes immediately beyond the narrow consideration of material gain (along the lines of the traditional tributary system) and goes on to mutual cultural appreciation and learning. The authors are not at pains to discuss what this learning might look like or how the "good 
life" from one state's point of view becomes revised on the basis of interaction with the other state, obviously in the direction of weak to strong being the more striking example.

And this is a major point. Apart from an authoritative tone that is perhaps not very warranted, the book's many topics-hierarchies between intimates, citizens, states, humans and animals, humans and machines-each truly demands a book of its own, and each contribution could be very powerful. The strongest section, meaning it is well researched and documented and clearly explained, is undoubtedly the comparison of political power and its furtherance through meritocratic versus democratic systems, a subject that the authors tackle with substance and subtlety but that certainly could be expanded to include more examples and suggested applications. Similarly, the discussion of ritual in Chapter 3, "Hierarchy between States," comes something closer to envisioning how to implement the ideal of a just hierarchy in the global-political context. The key philosopher in this part of their discussion is Xunzi 荀子 [313-238 в СE], one of the three major Confucian thinkers, specifically his ideas about the function of ritual among contending states. Ritual [ $l i$ 禮] is arguably the key concept in the Confucian understanding of how humans should interact generally, the social practice that allows us to rise above base self-interest and realize the benefits of mutual understanding and reliance most substantially. Xunzi's writings have notable implications for hierarchical relationships, in which rulers of stronger states, who could, of course, dispense with all pleasantries and simply plunder and subjugate the populace to achieve their aims, instead, take a different approach based on ritual. When viewed specifically in terms of strong reciprocal connections,

Rituals such as common birth, marriage, and burial practices also have the effect of including the poor and marginalized as part of the society's culture and common understandings. (p. 121)

Common or shared understanding is of major importance and, in practice, would lead to what Xunzi calls "humane authority" [wang 王], a condition in which strong states renounce strong-arm tactics [ $b a$ 霸] for winning the hearts of people near and far with the proper use of rituals. Again, this falls into the tianxia discussion mentioned above, which is why Bell and Wang advocate a revision to Zhao Tingyang's approach, such that the geographical parameters set by this "all under heaven" is now "all under heaven" only in East Asia, where one could imagine that rituals could be instated and ideally strong but even in some cases necessarily weak reciprocities establish a solid, stable approach to interstate dynamics. There are two problems with this picture: Bell and Wang 
address the first - the fact that US interests, in particular the US military presence, makes such a realignment unlikely in the short term — and the second (which they do not) is that this new East Asian tianxia bears unfortunate similarity to the Greater East Asian Co-Prosperity Sphere [dai toya kyoeiken 大東 亞共榮圈] envisioned by Japan during World War II, a period when a strongweak relationship was expressed in the form of brutality, mass killings, and systematic rape. Although Bell and Wang allow that other East Asian states are likely to be suspicious of China's intentions in this regard, I think that is, at best, an understatement.

But again, these are rapidly changing times, and the US vested interests in the region in particular may be shifting, which is to say in essence that the "strong-weak" dichotomy positioning the United States at the top might itself be undergoing basic inversion. The trade war, which Bell and Wang do not discuss, or the antics of President Donald Trump, which they mention six separate times, are already essential cases in point. That is even before CoviD-19 emerged, perhaps the most spectacular example of what happens when nonhierarchical systems, just or unjust, attempt to meet challenges to which they have no meaningful response. In other words, now is the time to be seriously considering the perspectives offered by Bell and Wang offer in this book, and, if for no other reason, it is important to read it.

\author{
Paul Manfredi \\ Department of Languages \& Literatures, Pacific Lutheran University, \\ Tacoma, WA, USA \\ manfredi@plu.edu
}

\title{
Work Cited
}

Bell, Daniel. China's New Confucianism: Politics and Everyday Life in a Changing Society. Princeton: Princeton University Press, 2008. 